\title{
Prevalence of Canine Babesiosis in Jos South Local Government Area of Plateau State
}

\author{
Moses Ojonugwa Oguche*, Israel Joshua Barde, Mayowa Peter Olabode, Waziri Ibrahim Anjili, \\ Victor Haruna, Daniel Apeh, Samson Mark, Joshua Bako Mafulul, Khalin Suleiman Wujat
}

National Veterinary Research Institute, Vom, Plateau State, Nigeria

Email: *mosimo4all@gmail.com

How to cite this paper: Oguche, M.O., Barde, I.J., Olabode, M.P., Anjili, W.I., Haruna, V., Apeh, D., Mark, S., Mafulul, J.B. and Wujat, K.S. (2020) Prevalence of Canine Babesiosis in Jos South Local Government Area of Plateau State. Open Access Library Journal, 7: e6401. https://doi.org/10.4236/oalib.1106401

Received: May 7, 2020

Accepted: June 8, 2020

Published: June 11, 2020

Copyright $\odot 2020$ by author(s) and Open Access Library Inc.

This work is licensed under the Creative Commons Attribution International License (CC BY 4.0).

http://creativecommons.org/licenses/by/4.0/

\section{(c) (i) Open Access}

\begin{abstract}
A study on the prevalence of canine babesiosis was carried out at Jos south Local Government Area of Plateau State, Nigeria, between May and June 2019. Giemsa stained blood smears of 150 dogs revealed that $20(13.33 \%)$ of the dogs were positive with Babesia canis. Among these, 63 (42.00\%) were puppies aged less than 6 months, and 87 (58.00\%) are adult dogs aged 9 months and above infected by the parasite. However, the age is found significantly correlated with Babesia canis as all the positives are in adults. P-value is $0.01(<0.01)$. Linear regression revealed that age is able to explain $26.9 \%$ of the variation observed in the lab results (prevalence of Babesia canis) at $\mathrm{P}$-value of $<0.01$. In bitches the infection rate was higher $(8.00 \%)$ than male dogs $(5.33 \%)$ and statistically not significant association $(p>0.05)$ was observed. The parasite was encountered in 13 (8.67\%) out of 60 local breeds and 7 (4.67\%) out of 90 exotic breeds. The breed-related prevalence rates were also not significantly different $(\mathrm{p}>0.05)$. Babesia canis was the only specie identified in the study area. The public health implications of the findings are discussed.
\end{abstract}

\section{Subject Areas \\ Parasitology, Veterinary Medicine, Zoology}

\section{Keywords}

Prevalence, Canine Babesiosis, Jos South, Public Health

\section{Introduction}

Babesia is a genius of parasite protozoan which affects several vertebrates, including humans. Babesiosis is the disease caused by the Babesia parasite, which is most common among dogs, cattle, horses and rodents [1]. 
All members of Babesiosis exhibit two life cycles: one in invertebrate host and the other vertebrate host; most common symptoms include fever, anemia, fatigue, aches, chills, hemoglobinuria and possibly death. Babesiosis is also known as Texas cattle fever among bovine. Humans may be subjected to the parasite through cattle Babesia bovis or rodents' Babesia microti; however only few cases are reported each year, usually among the elderly or those with compromised immune system [2].

These parasites are commonly called piroplasms because of the pear-shaped form found within infected Red Blood Cells. Babesia infections in human are infrequent and occur in limited geographic locations. Disease manifestations range from a symptomatic infection in healthy individuals to severe illness and death in those who are splenic, elderly, or immune compromised [3]. There are more than 70 species worldwide in the genius Babesia, with human infection largely due to the rodent strain. B. microtic is found only in the United States and the cattle strains Babesia divergence and Babesia bovis are found only in Europe [4].

Babesiosis is a zoonotic disease and required transmission from all animal's reservoir to humans via ticks' vector. In the northeastern United States, the block legged deer tick Ixodes scapularies, also called the Ixodes damminin. The incubation period in transfusion associated with the disease appears to be $6-9$ weeks; transplacental transmission has occurred rarely [5].

Parasites of this genus are primarily transmitted through tick bites and as such can infect a wide variety of domestic and wild animals as well as humans [6] [7]. Not surprisingly, dogs are one of Babesia spp.'s many targets, with various species of Babesia infecting canines and causing canine babesiosis [8]. Hard ticks are the main vectors for Babesia spp.; within the tick, Babesia spp. may be attached in one place or move around on the body of their host; other may spend part of their life on the skin as some are unable to move separately away from the host [9].

Babesia species comprises two main species, B.canis and B. gibsoni. Based on their size $B$. canis is a large piroplasm $(4-5 \mu \mathrm{m})$, which usually occurs as a single pear shaped piroplasm or in pairs of merozoites divided by binary fission within the erythrocyte, the basic difference in the geographical distribution, the vector specificity and antigenic properties. [10] recognized that large canine piroplasm is subdivided into three species namely, $B$.canis transmitted by Dermacentor reticulations (in Europe), B. vogel, transmitted by Rhipicephalus sanguineus (in tropical and sub-tropical region) and B. rossi associated with dogs in Asia, North America, Northern and eastern, Africa [11]. It is a small parasite that commonly appears as individual ring form or pyriform bodies ranging between 1.0 and 2.5 $\mu \mathrm{m}$ in size [12]. Clinically, canine babesiosis had been found to result in a wide range of presentation from subclinical disease to a serious illness characterized by fever, pallor, jaundice, splenomegaly, weakness and collapse associated with intra and extra vascular hemolysis, hypoxic injury, systemic inflammation, thrombocytopenia and hemoglobinuria [5].

As far as the diagnosis of canine babesiosis is concerned direct microscopic 
examination of the stained blood smear is the most commonly used method due to simple, easy, feasible and cost-effective diagnosis method but not necessary to detect parasite in dogs with unapparent or chronic infections since the level of parasitism is very low [13].

As regards to the serological method, indirect fluorescent antibody (IFAT) and enzymes linked immunosorbent assay (ELISA) for B. gibsoni parasite are considered to be highly sensitive but only moderate specific because of antigenic cross-reaction to $B$. canis [14]. Therefore, the development of highly specific and sensitive system for diagnosis of canine babesiosis is still awaited. In this regard, recent advances in molecular biology techniques like polymerase chain reaction (PCR) have made it possible to detect and identify babesiosis with greater sensitivity and specificity than traditional method [4] [15].

Regarding Nigerian scenario, though there are sporadic reports of canine babesiosis base on conventional diagnosis [16]. The true status of canine babesiosis is still not clear barring a few reports employ the PCR based assays. Furthermore, molecular detection of canine babesiosis has not yet been employed in part of Nigeria so the present work was carried out to know the prevalence of canine babesiosis in part of Jos South through light microscopy.

\section{Materials and Methods}

\subsection{Study Area}

Jos South is a Local Government Area in Plateau State, Nigeria. It houses the Governor's office in Rayfield and can thus be described as the de facto capital of plateau state. Its headquarters is located in Bukuru town on $9^{\circ} 48^{\prime} 00^{\prime \prime} \mathrm{N} 8^{\circ} 52^{\prime} 00^{\prime \prime E}$. It has an area of $510 \mathrm{~km}^{2}$ and a population of 306,716 at the 2006 census [17].

\subsection{Sample Collection}

One hundred and fifty blood samples were collected from seven breeds of dogs which are Alsatian, Bull Mastiff, Caucasian, Montreal, Lassa, and Rottweiler, both male and female from different age range, processed and examined under light microscope. The samples were collected aseptically in which $5 \mathrm{ml}$ of the blood were collected from the cephalic vein of each dog and was introduced into the ethylenediamine tetra acetic acid (EDTA) bottles from randomly selected dogs. After seeking permission from dog owners at different house hold and some from veterinary clinics within Jos South Local Government Area of Plateau State from May to June (period of 2 months) 2019. The needles were gently removed followed by gently/slowly sliding the samples to mixed properly with the anticoagulant, each sample specimens were properly labeled and recorded base on breed, sex, and age of the animals before taking to parasitology division of National Veterinary Research Institute (N.V.R.I) Vom, for analysis.

\subsection{Laboratory Processing of Samples}

Historically, Babesia infection in dogs was identified based on the morphologic appearance of the parasite in the erythrocyte; thus, microscopic evaluation for 
detecting intraerythrocytic parasites in Giemsa or Wright's stained blood smears remains the simplest, most accessible and reasonably sensitive especially during acute infections. Differentiation between large and small piroplasms is also relatively simple. Moreover, in many parts of the developing world where babesiosis is endemic, microscopy is still the only viable available option. The likelihood of spotting a piroplasm increases with proper sampling technique, namely: sampling from capillary beds (ear tip, toe nail) or examination of cells from beneath the buffy coat of a haematocrit tube [18]. The collected blood samples were utilized immediately for the preparation of thin blood smear, each blood was air dry fix on a fixed absolute methanol for 2 - 5 minutes' air dry and the blood were flooded with dilute Giemsa stain as described by Adam et al. [19] and hematocrit centrifugation technique (HCT) as described by Woo [20].

The pack cell volume (PCV) was determined according to standard procedure and the buffy coat examined for motile blood parasite for each sample using standard micro capillary method respectively [21].

\subsection{Data Analysis}

Data was collected and analyzed by using ANOVA to determine prevalence and association among different risk factors responsible for occurrence of canine babesiosis. Results were also presented in tables.

\section{Results}

Wet blood films show no motile haemoparasite while thin blood smear techniques showed the presence of only Babesia spp. in 20 dogs while 130 dogs were normal. Table 1 shows the total number of blood samples that reveal positive for babesia infection while rest of samples were negative for Babesia canis. The total number of positive and negative were (13.33\%) and (86.67\%) respectively. Table 2 shows that age is significantly correlated with Babesia canis as all the positives cases were recorded in adults. P-value is $0.01(<0.01)$. Linear regression revealed that age is able to explain $26.9 \%$ of the variation observed in the lab results (prevalence of babesia canis) at $\mathrm{P}$-value of $<0.01$. In Table 3 , the distribution of those infected breeds of dogs with Babesia canis shows that it is not prevalent within a particular age and breeds. The prevalence was higher in local breed (21.66\%) than in foreign breeds (7.77\%). It affects all sexes and irrespective of the breed. Babesia canis was the only specie identified in the study area.

\section{Discussion}

The results revealed that babesiosis is highly prevalent among all age groups, sex and breeds (Tables 2-4).

Table 1. Total number of bloods examined and number of blood sample positive and negative for babesiosis.

\begin{tabular}{ccccc}
\hline Total sample & No of sample (+) & No of sample $(-)$ & \% positive & \% Negative \\
\hline 150 & 20 & 130 & 13.33 & 86.67 \\
\hline
\end{tabular}


Table 2. Total number of samples examined based on age for canine babesiosis.

\begin{tabular}{cccccc}
\hline Age & Total sample & No of sample (+) & No of sample (-) & \% positive & \% Negative \\
\hline Puppies & 63 & 2 & 61 & 3.17 & 96.82 \\
Adult & 87 & 18 & 69 & 20.68 & 79.31 \\
\hline
\end{tabular}

All the positives are in adults. P-value is $0.01(<0.01)$.

Table 3. Total number of breed related sample examined for canine babesiosis.

\begin{tabular}{cccccc}
\hline Breeds & Total sample & No of sample (+) & No of sample (-) & \% positive & \% Negative \\
\hline Foreign & 90 & 7 & 83 & 7.77 & 92.22 \\
Local & 60 & 13 & 47 & 21.66 & 78.33 \\
\hline
\end{tabular}

Table 4. Total number of samples examined based on sex for canine babesiosis.

\begin{tabular}{cccccc}
\hline Sex & Total sample & No of sample $(+)$ & No of sample $(-)$ & \% positive & \% Negative \\
\hline Male & 65 & 8 & 57 & 12.30 & 87.69 \\
Female & 85 & 12 & 73 & 14.11 & 85.88 \\
\hline
\end{tabular}

The prevalence of canine babesiosis among breeds reveals that local breeds of dogs were more infected with babesiosis than the exotic, this could be due to lack of medical attention and poor management adopted by the breeders and tick bathing in local breeds of dogs as affirmed by Kamani et al. [22].

Also, the distribution of babesiosis by sex revealed that female dogs had the highest risk of the babesiosis base on the prevalence of this study. This could be attributed to their habits of roaming about for search of food for their puppies and as a result exposes them to the tick vectors Rhipicephalus sanguineus that transmit the Babesia canis [23].

The result also established that the prevalence of canine babesiosis was not too high in puppies as at the period of this study; probably because they are being confined and not allowed to roam about thereby leading to less risk of exposure to tick vectors.

As far as elevation of various risk factors is concern for canine babesiosis, several authors have observed the prevalence of the haemo-protozoan to be the highest in female dogs [24]. In terms of sex of the host, from the data obtained in the current study, it can be concluded that the PCV recorded no statistically significant difference $(>0.05)$ in the prevalence of the disease among males and females' dog. This result is incongruous with [25].

In seasonal Prevalence of the disease, the disease was more prevalent in winter, probably the reason behind this trend may be correlated to the seasonal activities of the brown dog ticks Rhipicephalus sanguineus which is more abundant in rainy period thus resulting to higher incidence of babesia canis infection as reported by Soulsby [21].

\section{Conclusions}

The findings of this study reveal the prevalence of canine babesiosis which is 
transmitted through the bite of ticks in the study area. Babesia canis was the only specie identified in the study area. Therefore, dog breeders and dog keepers are advised to always keep their dogs in a clean environment and restrict their movement especially in winter season free from ticks which transmit the disease. This can be done by regular tick bathing of dogs, cleaning of surroundings bushes and regular washing and spraying of kennels with acaricides. Dog breeders and keepers should ensure trimming of bushes and grasses where ticks are a serious problem. Dirt, wet and moist environment which enhances the survival of the tick's vectors that transmitted the babesiosis should be controlled and avoided.

Dog owners and breeders should always make it a point of duty to visit the veterinary clinic regularly for routine examination of their dogs for treatment against tick infestation and other related blood parasites.

Before breeding, the dog should be properly examined for parasite. Parasitological test should be carried out and proper treatment against tick borne diseases undertaken, because bitches tested positive are at high risk of possible abortion.

Varieties of drugs are available to treat Babesia canis with variable success. Imidocarb dipropionate is used mostly as a drug of choice and diminazene aceturate for successful treatment of canine babesiosis.

Dog owners and breeders are advised to maintain regular de-ticking of dogs. Homemade or self-medication should be avoided by dog owners and breeders.

\section{Conflicts of Interest}

The authors declare no conflicts of interest regarding the publication of this paper.

\section{References}

[1] Macintire, D.K. and Boozer, A.L. (2003) Canine Babesiosis. Veterinary Clinics of North America: Small Animal Practice, 33, 885-904. https://doi.org/10.1016/S0195-5616(03)00039-1

[2] Cleveland, W.C. and Coles, E.H. (2005) An Overview of Canine Babesiosis. College of Veterinary Medicine, The University of Georgia, Athens, GA, 40-141.

[3] Vannier, E. and Kraus, J.P. (2012) Human Babesiosis. The New England Journal of Medicine, 366, 2397-2407. https://doi.org/10.1056/NEJMra1202018

[4] Jefferies, W., Dan, U.A., Jeph, E.H. and Mayer, Y. (2008) PCR-RFLP for the Detection and Differentiation of the Canine Piroplasm Species and Its Use with Filter Paper-Based Technologies. Veterinary Parasitology, 144, 20-27.

[5] Irwin, P.J. (2009) Canine Babesiosis: From Molecular Taxonomy to Control. Parasite and Vectors, 2, Article No. S4. https://doi.org/10.1186/1756-3305-2-S1-S4

[6] Gorenflot, A., Moubri, K., Précigout, E., Carcy, B. and Schetters, T.P. (1998) Human Babesiosis. Annals of Tropical Medicine \& Parasitology, 92, 489-501.

https://doi.org/10.1080/00034983.1998.11813307

[7] Gray, J.S. (2006) Identity of the Causal Agents of Human Babesiosis in Europe. International Journal of Medical Microbiology, 296, 131-136. 
https://doi.org/10.1016/j.ijmm.2006.01.029

[8] Shaw, S.E., Day, M.J. and Birtles, R.J. (2001) Tick-Borne Infectious Diseases of Dogs. Trends in Parasitology, 17, 74-80. https://doi.org/10.1016/S1471-4922(00)01856-0

[9] Bell, J.M. and Palmer, S.R. (1988) The Zoonosis: Infection Transmitted from Animal and Man. 1st Edition, Edward Amoid, London, 173-174.

[10] Uilenberg, G., Franssen, F.F.J., Perie, N.M. and Spanjer, A.A.M. (1989) Three Groups of Babesia canis Distinguished and a Proposal for Nomenclature. Veterinary Quarterly, 11, 33-40. https://doi.org/10.1080/01652176.1989.9694194

[11] Hauschild, S., Shayan, P. and Schein, E. (1995) Characterization and Comparison of Merozoite Antigens of Different Babesia canis Isolated by Serological and Immunological Investigations. Parasitology Research, 81, 638-642. https://doi.org/10.1007/BF00931839

[12] Conrad, P.A., Thomford, J., Yamane, I., et al. (1991) Hemolytic Anemia Caused by Babesia Gibsoni Infection in Dogs. Journal of the American Veterinary Medical Association, 199, 601-605.

[13] Caccio, S.M., Antunovic, B., Moretti, A., et al. (2007) Molecular Characterization of Babesia canis and Babesia canis vogeli from Natural Infected European Dogs. Veterinary Parasitology, 106, 285-292. https://doi.org/10.1016/S0304-4017(02)00112-7

[14] Adachi, K., Tateshi, M., Horii, Y., Nagotomo, H., Shimizu, T. and Makimuru, S. (1994) Reactivity of Serum Anti-Erythrocyte Membrane Antibody in Babesia gibso$n i$-Infected Dogs. Journal of Veterinary Medical Science, 56, 997-999. https://doi.org/10.1292/jvms.56.997

[15] Jefferies, R., Ryan, U.M., Muhlnikel, C.J. and Irwin, P.J. (2003) Two Species of Canine Babesia in Australia: Detection and Characterization by PCR. Journal of Parasitology, 89, 409-412. https://doi.org/10.1645/0022-3395(2003)089[0409:TSOCBI]2.0.CO;2

[16] Sundar, N., balachandran, C. and Senthilvelan, A. (2004) Incidence of Babesia Gibsoni Infection in Dogs in Tamil Nadu. Journal of Parasilogy, 18, 79-80.

[17] Ministry of Agriculture and Natural Resources (MANR) (2008) Plateau State, Avian Influenza Control Office. Quarterly Bulletin, 1, 5-35.

[18] Poonam, V. and Nandini, M.K. (2019) Overview of Canine Babesiosis. Journal of Veterinary Parasitology, 141, 18-29.

[19] Adams, K.M.G., Paul, J. and Zaman, V. (1971) Medical and Veterinary Protozoology an Illustrated Guide. Church Livingstone, Edinburgh and London, 170-173.

[20] Woo, P.T.K. (1989) The Hematocrit Centrifuge Technique for the Detection of Trypanosome in Blood. Canadian Journal of Zoology, 47, 921-923. https://doi.org/10.1139/z69-150

[21] Soulsby, E.J.L. (2005) Helminthes, Arthropods and Protozoa of Domesticated Animals. 7th Edition, London, UK, 101-134.

[22] Kamani, J., Weka, P.R. and Gbise, S.D. (2011) Parasitic Cause of Anaemia in Dogs in VOM, Nigeria. International Journal for Agro Veterinary and Medical Science, 5, 283-289.

[23] Okoli, I.C., Okoli, C.G. and Opera, M. (2006) Environment and Multi-Host Infestation of the Brown Dog Tick, Rhipicephalus sanguineus in Owerri, South-East Nigeria-A Case Report. Veterinarski Arhiv, 76, 93-100.

[24] Abdullahi, S.U., Mohammed, A.R., Trimnelly, A., Sannusi, R. and Alafiatayo, R. (1990) Clinical and Haematological Findings in 70 Naturally Occurring Cases of 
Canine Babesiosis. Journal of Small Animal Practice, 31, 145-147.

https://doi.org/10.1111/j.1748-5827.1990.tb00750.x

[25] Amuta, E.U., Atu, B.O., Houmsou, R.S. and Ayashar, J.G. (2010) Rhipicephalus sanguineus Infestation and Babesia canis Infection among Domestic Dogs in Makurdi, Benue State, Nigeria. International Journal of Academic Research, 2, 170-172. 